\title{
Image Denoising In Gaussian And Impulsive Noise Based On Block Bidimensional Empirical Mode Decomposition
}

\author{
Faten BEN ARFIA \\ Computer Engineering System \\ design Laboratory (CES) \\ National Engineering School of \\ Sfax: Tunisia.
}

\author{
Mohamed BEN \\ MESSAOUD \\ Laboratory of Advanced \\ Technologies of medicine and \\ signals (UR-ATMS) \\ National Engineering School of \\ Sfax:Tunisia.
}

\author{
Mohamed ABID \\ Computer Engineering System \\ design Laboratory (CES) \\ National Engineering School of \\ Sfax: Tunisia.
}

\begin{abstract}
In this paper we develop an adaptive algorithm for decomposition and filtering of grayscales images. This method is highly adaptive decomposition image called Bidimensional Empirical Mode Decomposition (BEMD) based in blocks. This proposed approach decomposes image into a basis functions named Intrinsic Mode Function (IMF) and residue.

This method offers a good result in visual quality but it consumes an important execution time. To overcome this problem we propose a new approach using Block based BEMD method where the input image is subdivided into blocks. Then the conventional BEMD is applied on each of the four blocks separately. This proposed extended method gives a solution in reduction of execution time. This approach shows the good results in the field of image filtering. Denoised image is obtained by summing the residue and the filtered first IMFs (the detail) using a wavelet technique. Experimental results positively show that this proposed methodology removes Gaussian and Impulsive noises from the images.
\end{abstract}

\section{General Terms}

Image filtering, image decomposition.

\section{Keywords}

BEMD, BBEMD, IMF, Execution time, PSNR.

\section{INTRODUCTION}

Image decomposition is a common first step to the solution of many images processing such as medical image processing, image watermarking, image indexing ect. Generally, noise reduction is an essential part of image processing systems which can be ameliorated by the decomposition of the image [1][2]. An image is always affected by noise in its capture, acquisition and processing. The denoising process is used to improve the quality of corruption by a lot of noise due to the undesired conditions for image acquisition. Among the most common multiresolution methods of image decomposition include: spectral methods [2], Gabor functions [3] and wavelet transforms [4].

The traditional methods of decomposition are unable to remove noise effectively and produce a good visual quality for denoised images.
So the paper presents a decomposition of image based on the method of Multimodal Empirical Decomposition (EMD), introduced by Huang [5]. The approach provides a powerful tool for auto-adaptive multiscale analysis of non-stationary signals. Such technique has been successfully applied in many scientific problems. Its main advantage is the non dependence on an a priori basis set and it starts from the data itself to decompose the signals in spatial domain [6].

The BEMD approach is the two-dimensional version of the Empirical Mode Decomposition (EMD) which is based on the decomposition of nonlinear and non-stationary signals into intrinsic mode functions (IMFs) [7]. These functions are mono-component that has well-defined instantaneous frequencies. The BEMD has the same principle of EMD; it decomposes the input image into two-dimensional IMFs. This paper presents the modified BEMD based on the decomposition of the image blocks. This approach can solve the problem of the calculation time which has a handicap of BEMD approach for large images and presents an effective method to filter the images. This paper is organized as follows. Section 2 presents outlines the most significant of the Bidimensional Empirical Mode Decomposition (BEMD) and the details implementation of sifting process. Section 3 describes the modified BEMD based on the decomposition of the image blocks. . We describe the proposed noise reduction approach based in the BBEMD combining with the DWT technique in the section 4 . The section 5 shows the experimental results and the comparison with the methods used in this field. Finally, conclusions are made in Section 6.

\section{THE BIDIMENTIONNAL}

\section{EMPIRICAL MODE DECOMPOSITION}

Empirical Mode Decomposition (EMD) is first proposed by Huang [6] for the processing of non-stationary functions. The BEMD is the extension of the one dimensional approach with an adaptive decomposition of the images. It is based on the characterization of an image through its decomposition in intrinsic mode function (IMF) where the image can be decomposed into a redundant set of signals. This IMF function satisfying the following two conditions:

(a) The numbers of extrema and zero-crossings must be either equal or differ at most by one; 
(b) At any point, the mean value of the envelope defined by the local maxima and the envelope by the local minima is zero.

The Bidimensional sifting process is defined as follows [10]:

1. Initialization: $\mathrm{A}_{0}=\mathrm{r}$ (the residual) and $\mathrm{k}=1$ (index number of IMF)

2. Extraction of the $k^{\text {th }} I M F: I_{k}(m, n)$

a. Initialization: $E_{0}(m, n)=r_{k-1}(m, n)$ and $j=1$

b. Extract the local extrema of $E_{j-1}(m, n)$ (minima and maximum)

c. Interpolate the local extrema to construct the upper and the lower envelope respectively Envmin ${ }_{j-1}(m, n)$ and Envmax ${ }_{j-}$ ${ }_{1}(\mathrm{~m}, \mathrm{n})$

d. Calculate the average of the two envelopes:

$m_{j-1}(m, n)=\left(\operatorname{Env} \min _{\mathrm{j}-1}(\mathrm{~m}, \mathrm{n})+\operatorname{En} n v \max _{\mathrm{j}-1}(\mathrm{~m}, \mathrm{n})\right) / 2$

e. Update:

$$
E_{j}(m, n)=E_{j-1}(m, n)-m_{j-1}(m, n), \text { avec } \mathrm{j}=\mathrm{j}+1
$$

f. Calculate the stopping criterion

$$
S D(j)=\frac{1}{N} \sum_{t=0}^{T}\left[\frac{\left(E_{j-1}(m, n)-E_{j}(m, n)\right)^{2}}{E^{2}{ }_{j-1}(m, n)+\xi}\right]
$$

Where $\mathrm{x}$ is a term (low) to eliminate possible division by zero.

g. Decision: Repeat steps (b) to (f) until $\mathrm{SD}_{\mathrm{i}} \leq \mathrm{SD}_{\max }$, and then put $\mathrm{I}_{\mathrm{k}}(\mathrm{m}, \mathrm{n})=\mathrm{E}_{\mathrm{j}}(\mathrm{m}, \mathrm{n})$

3. Update residual

$$
r_{k}(m, n)=r_{k-1}(m, n)-I_{k}(m, n)
$$

Repeat steps 1-3 with $\mathrm{j}=\mathrm{j}+1$ until the number of extrema in $r_{j}$ is less than 2 .

The sum of all modes, added to the residual component reconstructs the original image [11]:

$$
A(m, n)=\sum_{k=1}^{K} I_{k}(m, n)+r(m, n), k \in N^{*}
$$

The Lena image presented in figure 1(a) is decomposed by BEMD algorithm. The correspondent IMF and residues are presented in figures 1 (b, c and d) and (e, f and g) respectively and finally the reconstructed image (h).

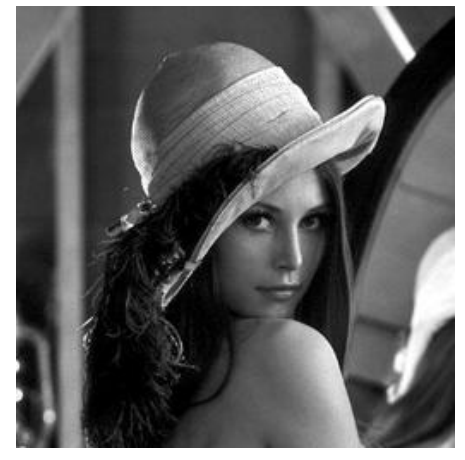

(a) original Image

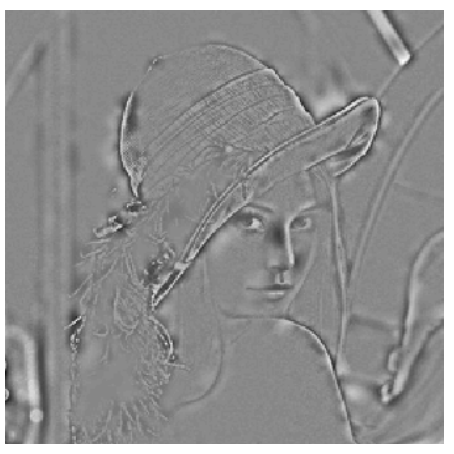

(b) IMF1

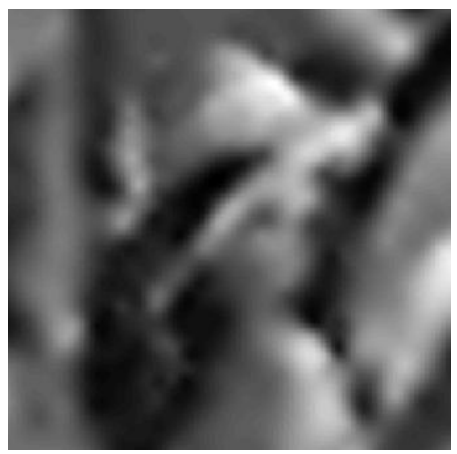

(d) IMF2

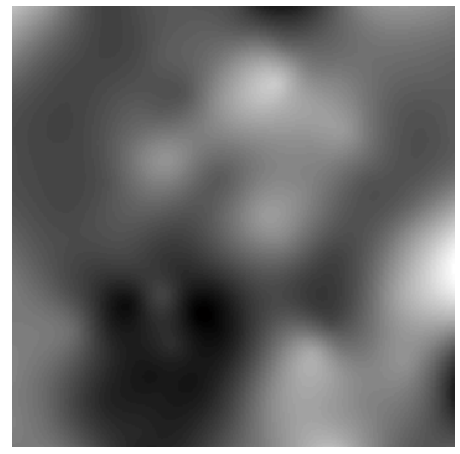

(f) IMF3

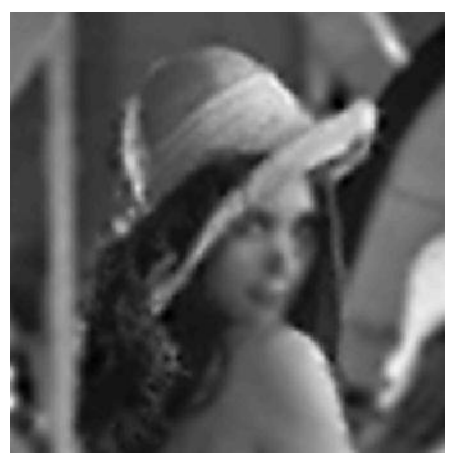

(c) residue 1

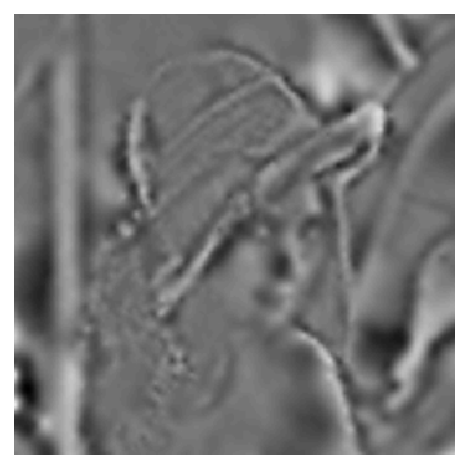

(e) residue 2

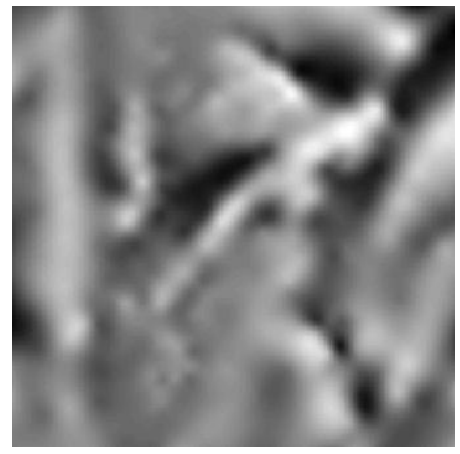

(g) residue 2 


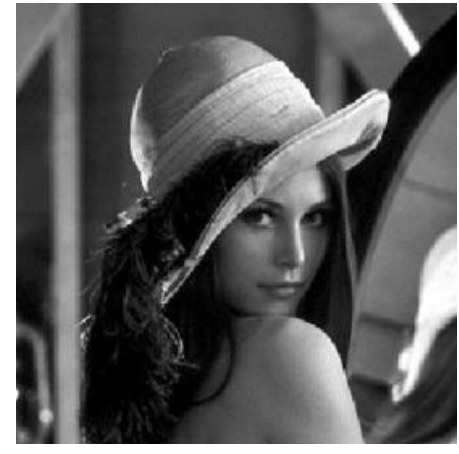

(h) Reconstructed image

Fig. 1: BEMD in three modes

\section{BIDIMENSIONAL EMPIRICAL MODE DECOMPOSITION BASED IN IMAGE BLOCKS}

The execution time presents a great handicap for the decomposition of large images. For solving this problem we propose a new approach based on the decomposition of original image into four blocks then we apply the BEMD algorithm in each block separately.

In the sifting algorithm we will processed each block separately to estimate minimum and maximum envelopes in the phase of RBF interpolation in small areas. For example, the decomposed image is treated with BEMD approach in four separated blocks, as shown in Figure 2. The proposed BEMD approach based image blocks is a good solution for reducing the execution time compared to BEMD (table 1).

Unfortunately, the significant gain in execution time of this approach can produce side effects (artifact).

Table 1: Values for execution time for the image decomposition using BEMD and BEMD based Block

\begin{tabular}{|c|c|l|l|l|l|}
\hline & \multicolumn{4}{|c|}{ Block-based BEMD } & BEMD \\
\hline & Bloc1 & Bloc2 & Bloc3 & Bloc4 & \\
\cline { 2 - 6 } & 7,066 & 6,191 & 7,971 & 8,99 & \\
\cline { 2 - 6 } $\begin{array}{c}\text { Execution } \\
\text { Time (s) }\end{array}$ & \multicolumn{4}{|c|}{30,218} & 64,64 \\
\cline { 2 - 6 } & \multicolumn{4}{|c|}{} & \\
\hline
\end{tabular}
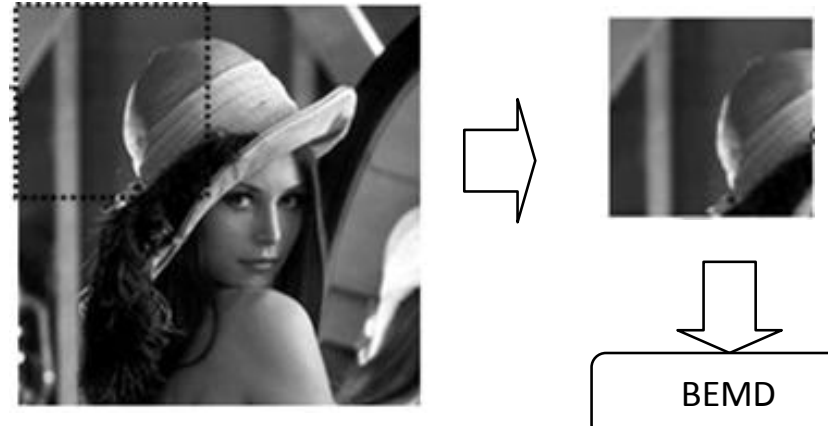

Original image

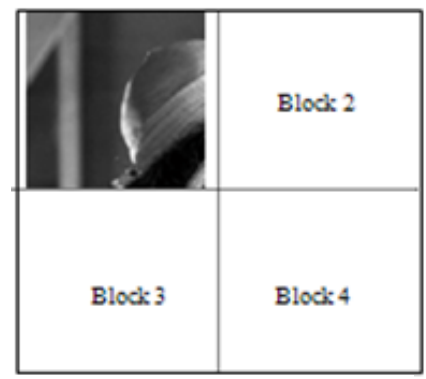

Reconstructed image

Fig. 2: The blocks based BEMD

\section{BEMD BASED IN BIDIMENTIONAL WAVELET TRANSFORM TECHNIQUE}

The BEMD approach is very effective in the denoising images field, but in the phase of decomposition it can be see that the first IMF, and probably the second, can contains the largest amount of noise.

By apply the wavelet transform to denoising the first and second IMF the PSNR criterion is calculated to improve the visual quality of the result image.

Several research projects have used the BEMD as filtering approach but it does not give good results in terms of visual quality for example the BEMD with the histogram. In this approach, they assumed that the noise is lower amplitude than the extrema points.

Therefore, the first IMF contains almost all the noise. So, to reduce the noise in a noisy image, they just reject all values lower than a specified threshold in the extrema points of the first IMF. They leave only the extremas points which are considered of significant points [12].

This threshold is determined from the histogram method of minimum and maximum extrema [13]. The proposed method is easy to implement but it causes a loss of information and poor visual quality in the denoised image as shown in figure 3. 


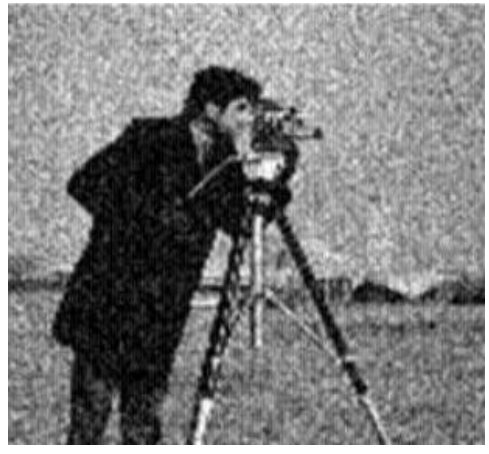

(a)

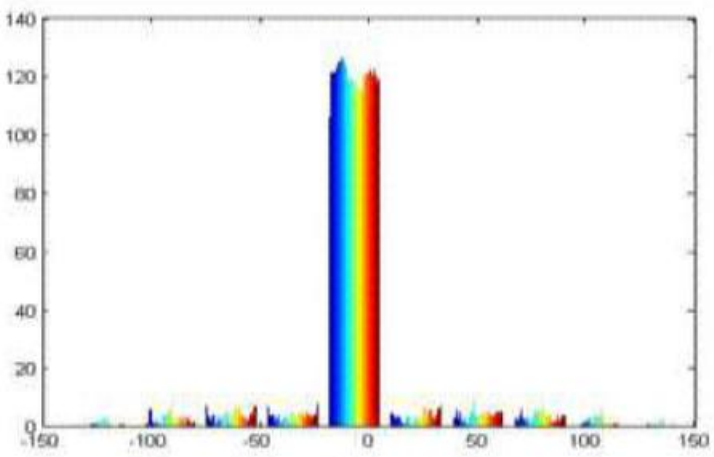

(b)

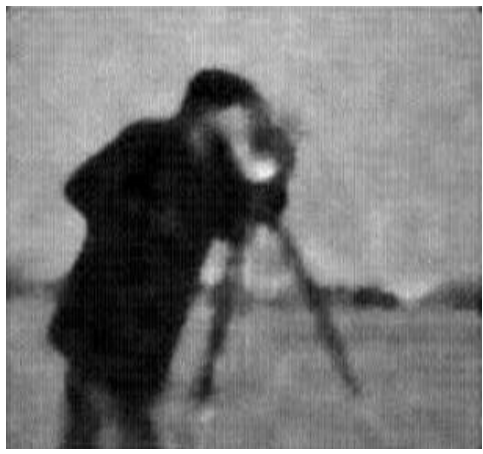

(c)

Fig.3. BEMD for denoising image: a) noisy image, b) histogram of the first IMF extremas, c) denoised image with BEMD

The BEMD in the image filtering consists in using the Wavelet Transform for filtering the first and the second IMF because the first IMF does not contain the amount of noise. In fact, this noise can also exist in the second IMF as opposed to the proposal which consider that the noise exists in the first IMF only [14]. This approach can improve visual quality and better performances than the traditional methods of image denoising. The description of the proposed approach is illustrated in the figure 4.

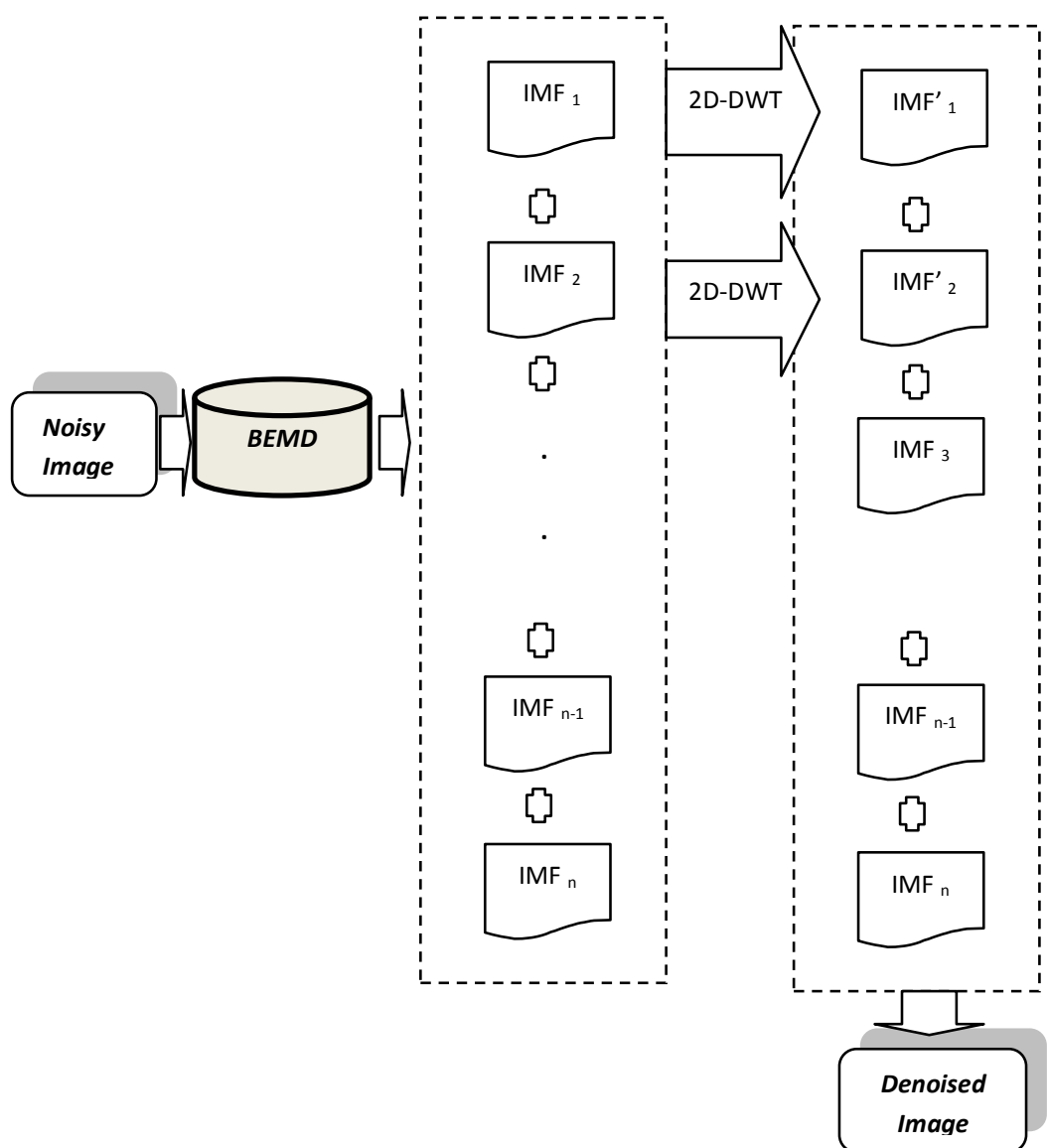

Fig. 4: Denoising image approach based in BEMD combined with DWT.

\section{EXPERIMENTS AND RESULTS}

In proposed approach we combine the BEMD and the DWT technique for image denoising. The proposed image denoising approach is applied on several natural grayscale images $(512 \times 512)$ that are contaminated by additive Gaussian noise with different noise levels $\sigma=5,10,15,20$ and 30 and the impulsive noise. Table 2 illustrates the denoising results of "cameramen.bmp" using BEMD with a single and 2 IMFs filtered with the Discrete Wavelet Transforms DWT.

Table 2. Performance of image denoising using BEMD with DWT for the Gaussian noise

\begin{tabular}{|c|c|c|c|c|}
\hline $\boldsymbol{\sigma}$ & \multicolumn{2}{|c|}{ BEMD (1 IMF) } & \multicolumn{2}{c|}{ BEMD (2 IMF) } \\
\hline & PSNR & MSE & PSNR & MSE \\
\hline 5 & 36,55 & 14,38 & 37,70 & 14,16 \\
\hline 10 & 31,80 & 41,94 & 32,97 & 39,28 \\
\hline 15 & 29,07 & 80,54 & 30,30 & 76,03 \\
\hline 20 & 27,21 & 123,48 & 28,50 & 119,90 \\
\hline 30 & 24,49 & 224,95 & 25,90 & 224,90 \\
\hline
\end{tabular}

The second column of table 2 shows that it is not efficient to filter to filter the first IMF to obtain an excellent result because the second IMF also can contain a quantity of noise. The filtering of this second IMF ameliorates the visual quality of the image in term of PSNR and MSE. 


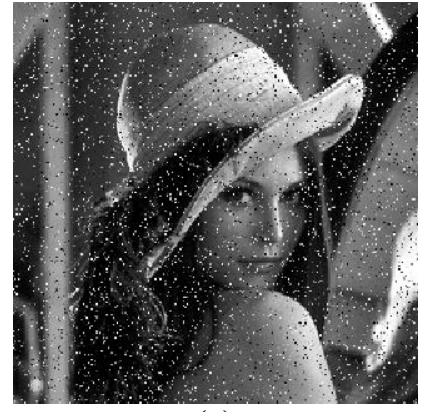

(a)

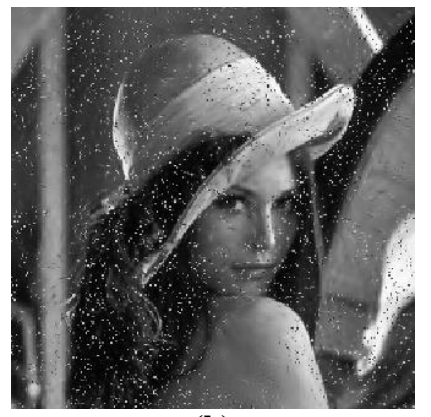

(b)
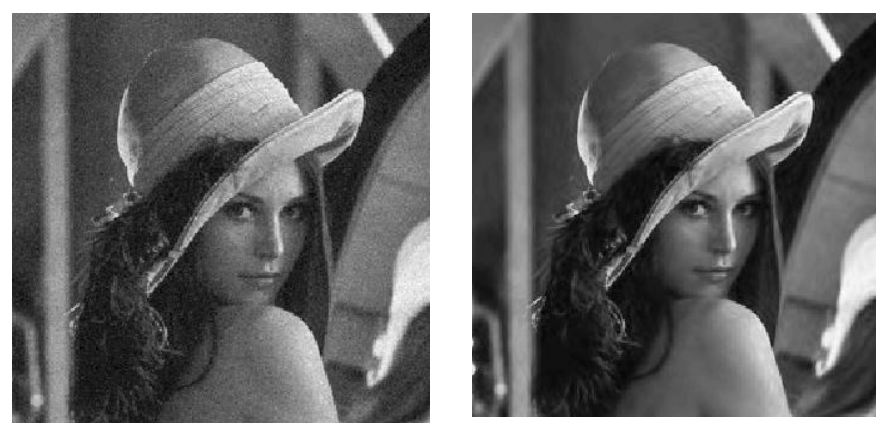

(c)

(d)

Fig. 5: Denoised images using BBEMD approach with 2 IMFs filtered: a) Noisy image with impulsive noise $10 \%$, b) Denoised image with BBEMD(2IMFs), c) Noisy image with Gaussian noise $\sigma=10, d)$ Denoised image with BBEMD(2IMFs) .

The figure 4 shows the performance of our approach and shows performance against to some non-linear adaptive filters of image denoising.

In comparison with traditional methods, table 3 and figure 4 illustrate the performance of the BEMD with DWT technique compared with the DWT and the median filter as nonlinear methods of denoising.

Table 3. Comparison results for denoising methods in impulsive noise in term of PSNR (db).

\begin{tabular}{|c|c|c|c|c|}
\hline $\boldsymbol{\sigma}$ & Noisy & BEMD & DWT & BBEMD(2IMFs) \\
\hline 10 & 17,72 & 20,6 & 24.68 & 24,7 \\
\hline 20 & 14,66 & 22.29 & 22,2 & 22,3 \\
\hline 30 & 12,92 & 20.48 & 20,36 & 20,53 \\
\hline 40 & 11,62 & 19.76 & 19,57 & 19,79 \\
\hline
\end{tabular}

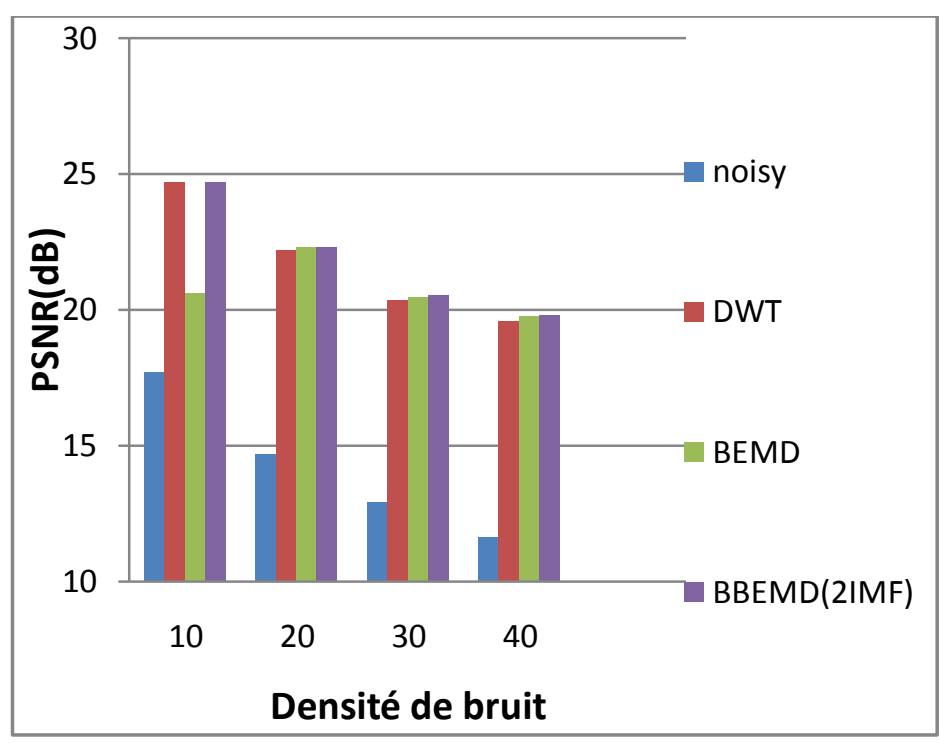

Fig. 6: The PSNR performance of the proposed method compared with the DWT and the BEMD approach in impulsive noise

Our approach proves also its performance for impulsive noise. We test this approach for an image with impulsive noise. The impulsive noise density $2 \%$ is added to the original image. The results in terms of PSNR and MSE are illustrated by table 4.

Table 4: Comparison results for denoising methods in term of PSNR (db) and MSE for impulsive noise (2\%)

\begin{tabular}{|c|c|c|c|}
\hline & $\begin{array}{c}\text { BEMD } \\
(1 \mathrm{IMF})\end{array}$ & $\begin{array}{c}\text { BEMD } \\
(2 \mathrm{IMF})\end{array}$ & DWT \\
\hline PSNR & 22,29 & 22,3 & 22,2 \\
\hline MSE & 391 & 369,90 & 393,48 \\
\hline
\end{tabular}

For concluding all results, the proposed approach gives better results to the levels of visual quality compared with the BEMD with the histogram approach as explained in paragraph 2 (figure 6).

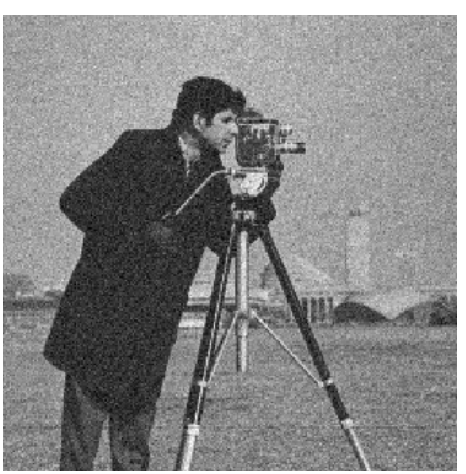

(a)

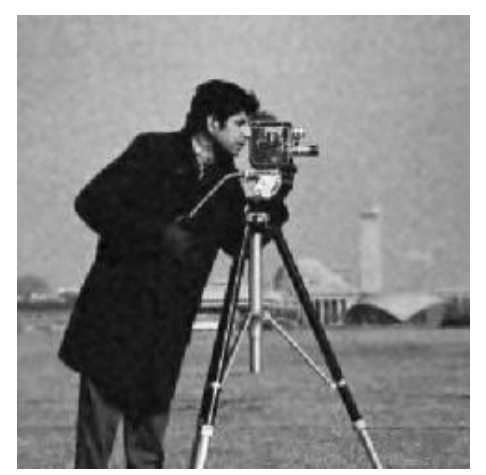

(b) 


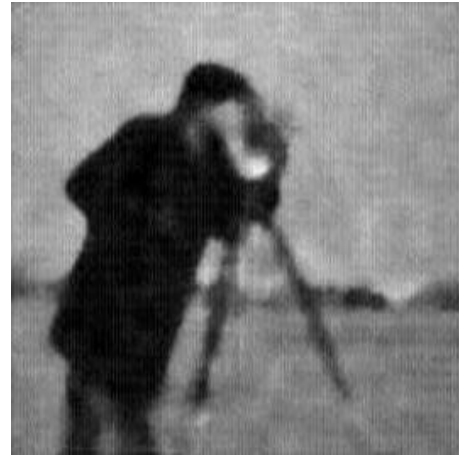

(c)

Fig. 6. (a) The noisy image with Gaussian noise $\sigma=20$, (b) denoised image with BEMD with DWT (2 IMF filtred), (c) BEMD with histogram.

\section{CONCLUSION}

The conventional BEMD approach is a powerful tool for denoising images. The disadvantage of this method is the important execution time in the real time applications. Our proposed idea is to subdivide the original image in bocks and to combine the BEMD with the 2D-DWT. On opposed of proposed methods wich use only the first one, this approach consists on the use of Wavelet Transform to filter the two first IMFs. Because the first IMF does not contain all of the noise, it can also be present in the second IMF. The proposed approach was tested on several grayscale natural images that are contaminated by an impulsive noise and a Gaussian additive one with different levels. The simulated results show the efficiency of the proposed approach wich can effectively improve the quality of the filtered image. The performance of denoising image, in term of execution time that can up to 50\% and visual quality that can arrive to $1,2 \%$ in terms of PSNR in comparison with traditional methods like DWT filtering technique.

\section{REFERENCES}

[1] LUKIN, V., OKTEM, R., N. PONOMARENKO and EGIAZARIAN, K., 2007 Image filtering based on discrete cosine transform, Telecommunications and Radio Engineering, 66, No 18, 1685-1701.

[2] DONOHO, D.L AND JOHNSTONE L.M., 1994 Ideal Spatial Adaptation via Wavelet Shrinkage, Biometrika vol.81, 425-455.

[3] MALLAT, S. G., 1989A theory for multiresolution signal decomposition: the wavelet representation, IEEE Trans. Pattern Analysis and Machine Intelligence, Vol 11, 674-693.
[4] P. PERONA AND J. MALIK, Scale-space and edge detection using anisotropic diffusion, IEEE Trans, Pattern Analysis and Machine Intelligence, Vol. 12, No. 7, pp.629-639, 1990.

[5] DAMERVAL, C. , MEIGNEN, S. AND PERRIER, V. 2005 A fast algorithm for Bidimensional EMD, IEEE Signal Processing Letters, Vol. 12, 701-704,.

[6] HUANG, NE., SHEN, Z., LONG, SR., WU, MC., SHIH, HH., ZHENG, Q., YEN, N-C., TUNG, CC. AND LIU, HH. 1998 The empirical mode decomposition and the Hilbert spectrum for non-linear and non-stationary time series analysis, Proc R Soc Land Ser A, 903-995.

[7] BEN ARFIA, F., BEN MESSAOUD, M. AND ABID, M. 2010 A New Image denoising Technique Combining the Empirical Mode Decomposition with a Wavelet Transform Technique 17th International Conference on Systems, Signals and Image Processing.

[8] NUNES, J. C., BOUAOUNE, Y., DELECHELlE, E., NIANG, O. AND BUNEL, PH. 2003 Image analysis by Bidimensional empirical mode decomposition, Image and Vision Computing.

[9] LINDERHED, 2002 A. 2D empirical mode decompositions - in the spirit of image compression, Proc. of SPIE, Vol. 4738, 1-8.

[10] SABRI, A., KAROUD, M., TAIRI, H. AND AARAB, 2008 A. Fast Bidimensional Empirical Mode Decomposition Based on an Adaptive Block Partitioning, International Journal of Computer Science and Network Security, VOL.8.

[11] LINDERHED, A., 2002 2D empirical mode decomposition in the spirit of image compression, Wavelet and ICA Applications IX, SPIE Proceedings, Orlando, 1-8.

[12] LINDERHED, A., 2009 Image Empirical Mode Decomposition: a New Tool for Image Processing, Advances in Adaptive Data Analysis, 265-294.

[13] ABDELOUAHED, S., 2009 Décomposition multimodale empirique bidimensionnelle : Améliorations et Applications. Theses, university of Sidi Mohammed Ben Abdellah Faculty of sciences Dhar el Mehraz Fes, Marooc.

[14] DEMIR， B. ， S. ERTUURK, M.K GUULLUU, Hyperspectral Image Classification Using Denoising of Intrinsic Mode Functions, Geoscience and Remote Sensing Letters, IEEE, Volume 8 Issue 2, 220 - 224 , 2011. 